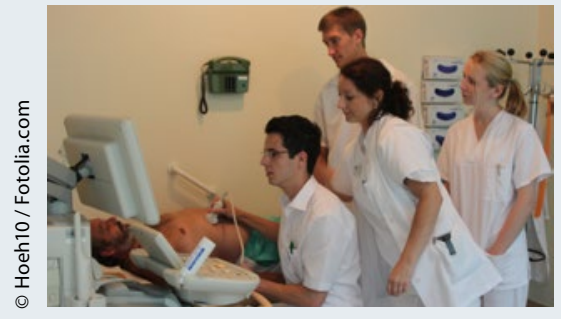

Studenten gegen stärkere Einbindung der Allgemeinmedizin

Die von der Bundesregierung im Masterplan Medizinstudium 2020 vorgesehene Stärkung des Faches Allgemeinmedizin im Medizinstudium trifft auf große Skepsis. $74 \%$ der 1.756 Medizinstudenten des Marburger Bundes halten eine stärkere Einbindung der Allgemeinmedizin in das Studium für nicht notwendig, so eine Erhebung. $86 \%$ lehnen die von der Deutschen Gesellschaft für Allgemeinmedizin und Familienmedizin erhobene Forderung nach einem Pflichtabschnitt Allgemeinmedizin im Praktischen Jahr ab. Matthias Wallenfels

\section{Arztbewertungsportale: BGH stärkt die Rechte von Ärzten}

Der BGH in Karlsruhe hat entschieden (VI ZR 34/15), dass Bewertungsportale auf Verlangen beispielsweise stichhaltige Belege darüber vorlegen müssen, ob ein Nutzer tatsächlich in der Praxis eines dort bewerteten Arztes oder Anwaltes war. Namen eines Bewerters müssen die Portale aber auch in Zukunft nicht herausrücken. Im konkreten Fall ging es um einen Zahnarzt, der sich im Portal jameda.de zu Unrecht schlecht bewertet sah.

Martin Wortmann

\section{IGeL: Patienten vertrauen ihren} Ärzten

Selbst wenn Kassenpatienten Bedenken gegen eine offerierte IGeL haben, vertraut etwa jeder Dritte von ihnen im Zweifelsfall auf die medizinische Kompetenz ihres Arztes. Das zeigt eine repräsentative Umfrage im Auftrag der Techniker Krankenkasse. Nach den Gründen befragt, warum sie sich doch für die Inanspruchnahme der Leistung entschieden hätten, führen $32 \%$ die ärztliche Kompetenz ins Feld. An zweiter Stelle mit 27,4\% rangiert die Auffassung, dass eine Diagnostik oder Therapie mehr nicht schaden könne. ich eine Videosprechstunde in die Praxisabläufe ein?" Vor allem sollten Ärzte mit der Umsetzung digitaler Prozesse nicht so lange warten, bis die Leistungen auch ausreichend honoriert werden. „Natürlich müssen digital erbrachte Leistungen auf die Dauer bezahlt werden. Aber darauf zu warten, welches Geld gezahlt werden wird, und erst dann zu investieren, wäre die falsche Haltung. Denn die Leistungen werden von immer mehr Patienten eingefordert", warnt Heßbrügge.

Nicht jede Fachgruppe bei den Ärzten müsse gleich schnell auf die Digitalisierung reagieren, räumt der Vordenker ein. „Eine Gynäkologin, die viele junge Mütter betreut, muss viel mehr damit rechnen, dass ihre Patientinnen erwarten, auch online Termine buchen zu können oder Befunde online abzurufen, als ein Orthopäde, der sich auf Reha-Patienten spezialisiert hat." Bei Sportmedizinern sehe es natürlich ebenfalls anders aus als bei Geriatern - wobei die auch schon wieder über den Kontakt mit den betreuenden Angehörigen oder auch mit Pflegeheimen Nachfragen nach digitalen Anwendungen gegenüberstehen und mit Kompetenz in Telemedizin punkten könnten.

Ein Arzt, der sich der neuen Technik eher langsam nähert, verliere sicher nicht auf einmal alle Patienten, aber es könne doch ein schleichender Prozess einsetzen, der verhindert, dass neue $\mathrm{Pa}$ tienten für die Praxis gewonnen werden. Wenn der Arzt keine Online-Terminbuchungen ermögliche, führt das bereits in manchen Patientenkreisen zu der Vermutung, „dass dann auch das Ultraschallgerät in der Praxis wohl schon etwas älter ist. Da passiert etwas, das für Ärzte hoch relevant ist, ohne dass im EBM Ziffern dafür gebaut sind“, warnt Heßbrügge.

Hauke Gerlof

\title{
Netzförderung kommt in den Regionen an
}

\section{Die vom Gesetzgeber in § 87b SGB V geforderte Anerkennung und Förderung von Arztnetzen macht gute Fortschritte. Diese Zwischenbilanz zieht die Agentur deutscher Arztnetze aus ihrer Nachforschungsarbeit zu Beginn des Jahres.}

$D^{i c}$ ie Agentur deutscher Arztnetze (ADA) hat im Februar erneut bei den KVen nachgehakt. Das Ergebnis: 15 KVen haben tatsächlich die Rahmenvorgaben der KBV zur Netzförderung umgesetzt und Kriterien zur Anerkennung von Netzen veröffentlicht. Dabei sind bundesweit bislang 33 Netze anerkannt worden. Die KV Niedersachsen hat zwar keine Richtlinie erlassen, fördert die Netze aber. Damit steht lediglich die KV Mecklenburg-Vorpommern noch gänzlich ohne Richtlinie und Förderung da.

Fördergelder erhalten die Netze in mittlerweile zehn Regionen. „Wobei deren Umfang von KV zu KV erheblich schwankt", so die ADA. So können Ärzteverbünde in Niedersachsen bis $\mathrm{zu}$ $50.000 €$ je Netz erhalten, in SchleswigHolstein und in Westfalen-Lippe sind es sogar bis zu $100.000 €$. Im Saarland hingegen sieht die Förderung lediglich bis zu $100 €$ je Arzt für die Teilnahme an bis zu zwei Qualitätszirkeln pro Quartal vor. Und in Sachsen-Anhalt können Netzärzte im Einzelfall eine Unterstützung von $1.000 €$ pro Netz beantragen. Gar keine finanzielle Förderung sehen bislang die KVen in Baden-Württemberg, Berlin, Brandenburg, Bremen, Hamburg, Mecklenburg-Vorpommern und Hessen vor. Wobei es in Hessen aber eine Förderrichtlinie des Sozialministeriums gibt, über die für neun Regionen eine Fördersumme von 1 Millionen $€$ auch für die Netzarbeit bereitstehen soll.

In Reaktion auf die Förderbilanz vertritt der Ärzteverband Medi Geno das Ansinnen, Arztnetze sollten lieber versuchen, unabhängig von der KV zu bleiben und Fördergelder eher in der Region bei Krankenkassen und Kommunen zu organisieren, denn diese profitierten letztlich auch von der besseren medizinischen Versorgung vor Ort.

Rebekka Höhl und Christoph Winnat 